\title{
Technè
}

La science au service de l'histoire de l'art et de la préservation des biens culturels

$44 \mid 2016$

Archives de l'humanité : les restes humains patrimonialisés

\section{La conservation-restauration des restes humains patrimonialisés : questions de déontologie dans le domaine français}

The conservation-restoration of human remains incorporated into cultural heritage: questions of deontology raised in France

\section{Noëlle Timbart}

\section{OpenEdition}

\section{Journals}

Édition électronique

URL : http://journals.openedition.org/techne/1154

DOI : 10.4000/techne.1154

ISSN : 2534-5168

\section{Éditeur}

C2RMF

\section{Édition imprimée}

Date de publication : 1 novembre 2016

Pagination : 96-102

ISBN : 978-2-7118-6339-6

ISSN : $1254-7867$

Référence électronique

Noëlle Timbart, «La conservation-restauration des restes humains patrimonialisés : questions de déontologie dans le domaine français », Technè [En ligne], 44 | 2016, mis en ligne le 19 décembre 2019, consulté le 23 juillet 2020. URL : http://journals.openedition.org/techne/1154 ; DOI : https://doi.org/ 10.4000/techne.1154

\section{(c) (i) (9)}

La revue Technè. La science au service de l'histoire de l'art et de la préservation des biens culturels est mise à disposition selon les termes de la Licence Creative Commons Attribution - Pas d'Utilisation Commerciale - Pas de Modification 4.0 International. 


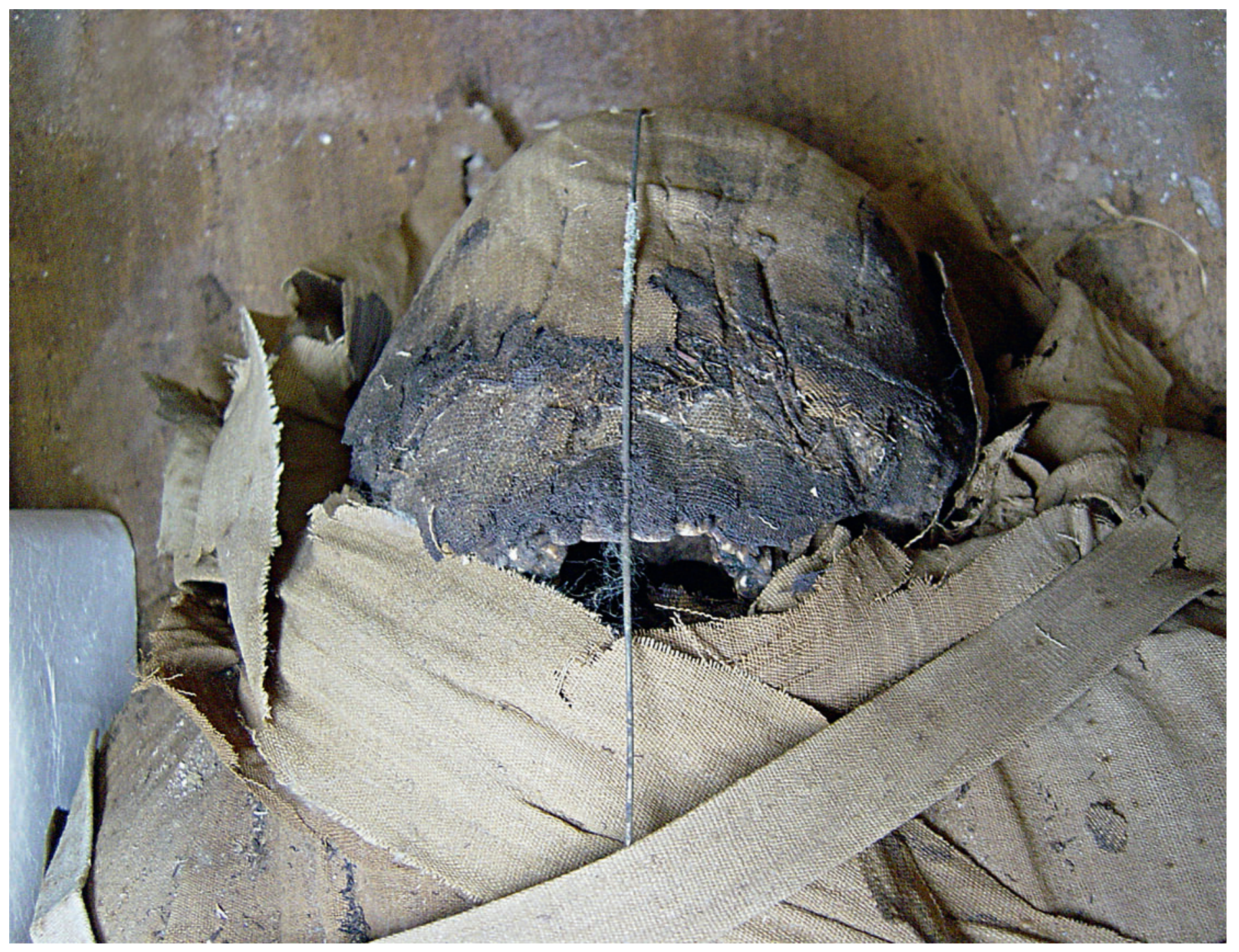

Fig. 1. Ancienne restauration : fil métallique utilisé pour maintenir la tête accrochée au corps. @ C2RMF/J. Marsac. 
Noëlle Timbart

\section{La conservation-restauration des restes humains patrimonialisés : questions de déontologie dans le domaine français}

The conservation-restoration of human remains incorporated into cultural heritage: questions of deontology raised in France

Résumé. Conserver et restaurer des restes humains patrimonialisés dans les musées soulève des questions d'ordre éthique, social et culturel. Les choix d'intervention sont délicats et posent la question des limites de lintervention. La conservation-restauration de ces collections s'appuie sur des doctrines et des principes qui guident les interventions, la déontologie. Or, pour les restes humains, il semblerait que la réflexion en France soit relativement récente. Cet article se propose de souligner les principes déontologiques mis en cuvre dans le cadre de traitements de conservationrestauration, les restes humains ne pouvant être considérés uniquement du point de vue matériel. Chaque intervention suppose une réflexion préalable faisant intervenir les différents acteurs concernés. La subjectivité et la sensibilité individuelle occupent une place prégnante, mais l'éthique demeure au cœur de l'intervention : le statut d'individu derrière le corps patrimonialisé ne doit pas être oublié.

Mots-clés. Restes humains patrimonialisés, conservationrestauration, déontologie, éthique, respect.

\begin{abstract}
Conserving and restoring human remains which are part of museum collections raises ethical, social and cultural questions. Any intervention involves delicate decisions and demands reflection on the extent to which one may intervene. The conservation-restoration of these collections relies on deontology: doctrines and principles that guide interventions. Where human remains are concerned, in France, such reflection seems to have been relatively recent. This article seeks to emphasize the deontological principles applied to conservation-restoration treatments, since human remains cannot be considered solely from a material point of view. Each intervention implies prior reflection from all the different concerned parties. Subjectivity and individual sensibility play a significant role, but ethics remain central to the intervention: the status of the individual to whom the body in the museum once belonged must not be forgotten.

Keywords. Human remains granted heritage status, conservation-restoration, deontology, ethics, respect.
\end{abstract}

Conserver des restes humains dans des collections muséales ne va pas de soi. Or le corps humain est présent au musée sous toutes ses formes (ossements, corps, organes, peaux, entier ou fragmentaire, desséché ou en fluide, etc.). Sa présence est le plus souvent liée à l'histoire de la constitution des collections, à partir des cabinets d'amateurs qui présentaient des restes humains dès le XVI ${ }^{\mathrm{e}}$ siècle. Ces collections ont ensuite acquis un statut scientifique dans une volonté encyclopédique de connaissance du monde et de l'Homme. Les conservateurs qui en ont aujourd'hui la charge se doivent de les conserver au même titre que les autres objets inscrits à l'inventaire afin d'en assurer la transmission. La conservation peut impliquer, dans le cadre d'exposition, de valorisation mais aussi d'amélioration de la lisibilité des collections, des opérations de conservation-restauration.

Mais comment concilier l'approche scientifique et les valeurs culturelles et sacrées qui sont attachées au corps ? Il y a une grande part de subjectivité et de multiples attitudes, notamment dans les pratiques muséales.
De plus, se posent les questions de l'éthique et de la déontologie ${ }^{1}$ de ces interventions. Conserver et restaurer la dépouille d'êtres humains constitue un domaine spécifique puisqu'il s'agit de matériel humain, par conséquent, particulièrement sensible et à fort impact émotionnel. Certains restes humains, tels les momies amérindiennes qui ont souvent la bouche ouverte, peuvent impressionner le public, de même, que les momies ou squelettes d'enfants, car ils renvoient à l'humain, au vécu et à la mort. Le corps est plus qu'un simple objet matériel. En outre, périssable de par sa nature même, le but de l'intervention sera donc de préserver et de prolonger un corps ou élément de corps qui est voué à disparaître à plus ou moins long terme.

\section{Mise en place récente d'une réflexion en France}

Si l'attitude des conservateurs du patrimoine a considérablement évolué ces dernières années sur ce sujet délicat, la réflexion, en France, sur la déontologie de la conservation-restauration de

Noëlle Timbart, conservateur du patrimoine chargé des Antiquités égyptiennes et orientales et des restes humains, département Restauration, C2RMF (noelle.timbart@culture.gouv.fr). 
ces collections semble relativement récente. Peu de documents abordent ce sujet spécifique. En 2006, Laure Cadot s'interrogeait dans son mémoire de Master 2 sur ce point et établissait un état des lieux de la question en France, soulignant la nécessité de la mise en place d'une réflexion à l'échelle nationale. Ces questions ont été relayées par la publication de son mémoire et par divers articles, notamment dans La Lettre de l'OCIM ${ }^{2}$.

En outre, plusieurs colloques et journées d'étude se sont tenus autour de la question de l'exposition du corps humain et de sa conservation dans des institutions muséales à la suite du symposium «Des collections anatomiques aux objets de culte : conservation et exposition des restes humains dans les musées ", organisé au musée du quai Branly en 2008, qui a amorcé la réflexion sur les aspects éthiques et déontologiques au sein de la communauté professionnelle ${ }^{3}$. D'autres colloques, tels « Exposer le corps humain : gestion, déontologie et questions juridiques" et «Les collections anatomiques : de la connaissance à la mise en valeur » organisés par l'INP en 2010 et 2012, témoignent du renforcement de l'intérêt pour la question. Néanmoins, la restauration proprement dite de ce type de collections n’a pas vraiment été abordée même si des orientations bibliographiques ont été données ou si une table ronde a été consacrée à ces problématiques.

De fait, les enjeux éthiques et les principes déontologiques mis en œuvre transparaissent dans plusieurs articles qui relatent des opérations ponctuelles de conservation-restauration. Celles-ci traduisent la prise de conscience des professionnels en charge de ces collections des enjeux entourant ces vestiges ${ }^{4}$. Et depuis décembre 2014, un groupe de travail ${ }^{5}$ constitué au sein de la Commission Scientifique Nationale des Collections est chargé de réfléchir plus particulièrement à ces collections.

En revanche, dans les pays anglo-saxons, et notamment en Grande-Bretagne, la réflexion s'avère beaucoup plus poussée et plus ancienne. Un document intitulé Guidance for the Care of Human Remains in Museums (Department for Culture, Media and Sport) a été rédigé en 2003-2004 sur la conservation des restes humains, suite à la constitution d'un groupe de travail sur les restes humains dès $2001^{6}$. Plus récemment, le numéro 197 de la revue du British Museum a, quant à lui, été consacré à la question des restes humains : Regarding the Dead: Human Remains in the British Museum (2014). Les questions éthiques et déontologiques relatives à la présence des restes humains dans les musées et les sujets liés à la conservation, à l'étude, au stockage, à l'accès, à la gestion et à l'exposition y sont abordés. Plusieurs règles et pratiques ont été mises en place dans les institutions muséales à la suite de la création de ce document. De même, le Human Remains: Guide for Museums and Academic Institutions de Cassman et Odegaard (2007) encadre les pratiques de conservation et d'exploitation scientifique spécifiques à ce domaine ${ }^{7}$.

\section{La déontologie de la restauration en France}

La restauration des biens culturels en France est définie par des codes nationaux (Code du Patrimoine notamment). Elle « consiste à intervenir directement sur des biens culturels endommagés ou détériorés dans le but d'en faciliter la lecture tout en respectant autant que possible leur intégrité esthétique, historique et physique ${ }^{8}$ " et implique les notions de compatibilité, de stabilité, de réversibilité et de lisibilité des interventions qui sont au cœur de tout traitement ${ }^{9}$. Toutefois, aucune section n'est spécifiquement dédiée aux restes humains.

Or, restaurer un reste humain n'est pas un geste anodin. La question de la conservation-restauration des restes humains est cruciale puisqu'elle implique de justifier la conservation de ces restes intrinsèquement voués à la destruction, ainsi que la manière de le faire avec les limites qui en découlent. Ainsi, la prise en compte de l'état de dégradation des corps est essentielle de même que celle du processus mis en œuvre dans leur préservation initiale (degré d'intentionnalité) ${ }^{10}$ selon les cultures dont ils sont issus.

Si on analyse les différentes restaurations menées en France dans ce domaine, il apparaît que ce sont essentiellement les codes de déontologie internationaux, tels que les codes de déontologie de l'ICOM (2006 et 2013) et le code éthique de l'ECCO (2003), qui servent de référence. C'est la déontologie de la profession de restaurateur qui est appliquée. Les règles du code de déontologie de l'ICOM (2006) ${ }^{11}$ énoncent que la conservation et l'exposition des restes humains, matériel culturel sensible, doivent se faire en accord avec les normes professionnelles et, lorsqu'ils sont connus, les intérêts et croyances de la communauté ou des groupes ethniques ou religieux d'origine. Mais les notions de respect et de dignité humaine doivent primer ${ }^{12}$. Ces préoccupations éthiques se sont particulièrement développées ces dernières années concernant les questions conservatoires liées aux restes humains. Elles transparaissent dans les usages, aussi bien dans le cadre des pratiques scientifiques que des pratiques muséales, affichant une démarche plus responsable et respectueuse et un souci de dignité. Ainsi, plusieurs institutions muséales ont entrepris de s'occuper de ces collections qui étaient remisées dans les réserves dans des conditions souvent peu optimales (cartons «fourre-tout », corps sous des bouches d'évacuations d'air, à la poussière, etc.), en leur assurant une étude, une présentation à l'intérieur de vitrines conçues spécifiquement à leur intention ou en réalisant des conditionnements adaptés, leur offrant des conditions de conservation adéquates.

Pendant longtemps, la restauration des restes humains reposait avant tout sur des traitements visant à pallier les problèmes de conservation des matériaux organiques fragiles qui les constituent. Les interventions portaient essentiellement sur des consolidations à base d'adhésif ou de plâtre, par l'adjonction de textiles, des bouchages pour combler les manques, des éléments métalliques (fig. 1) pour relier deux parties de corps ; l'emploi de vernis protecteurs ou de solutions à base d'arsenic pour traiter les infestations à l'instar de la momie andine du musée de l'Homme $\left(n^{\circ} 1369\right)^{13}$; et la mise 
en place de supports muséographiques plus ou moins intrusifs, telles que les potences utilisées pour la présentation des momies d'Amérique du Sud qui se caractérisent par leur position assise. Ces anciennes interventions et les manipulations qui y sont liées ont été à la source de nouvelles dégradations engendrant des altérations matérielles (usure de la peau, cassure du squelette, os brisés notamment), mais aussi des pertes d'informations scientifiques. Et l'aspect « humain » n'était pas pris en considération. Dans le cas des momies d'Amérique du Sud, nombre de soclages avec potences métalliques ont pu transpercer les corps du bassin jusqu'au sternum sans tenir compte du point de gravité ; ou des tiges métalliques ont pu être utilisées pour maintenir fixée la tête au corps, de façon très intrusive.

Aujourd'hui, toute intervention de restauration considère que le corps reste, après la mort, fortement déterminé par le respect dû à la personne (révision des lois de bioéthique, 2010). L'individu est considéré comme primordial dans le traitement : la dimension humaine est devenue un paramètre incontournable. Par conséquent, un soin plus particulier est accordé à l'aspect minimal de l'intervention qui préserve les informations dans le cadre de futures recherches qui pourront être menées sur les individus, permettant ainsi de conserver le potentiel informatif $\mathrm{du}$ corps. En effet, ces collections représentent un intérêt scientifique indéniable sur divers aspects d'une civilisation (croyances religieuses, pratiques funéraires, conditions de vie, etc. ${ }^{14}$ : les opérations de conservationrestauration doivent donc veiller à préserver les témoins de son histoire et de son usage. Ainsi, bien souvent, le traitement du corps se limite à un simple dépoussiérage et à une remise en connexion anatomique des os du squelette si nécessaire, quand celui-ci est accessible.

Par ailleurs, les avancées technologiques, à l'image du scanner aujourd'hui, permettront de compléter les données que ces corps véhiculent et qu'il n'est pas toujours possible de déterminer à l'heure actuelle. C'est pourquoi la recherche devrait être intégrée à l'acte de restauration proprement dit. Afin de favoriser les examens et analyses, il importe en outre de limiter au maximum les contaminations par des produits qui masqueraient des informations, ou les dégradations physico-chimiques (C) C2RMF/A. Chauvet. et toute intervention invasive qui empêcherait de percevoir certains détails pour garantir la conservation du potentiel informatif que représente le corps objet de l'étude et du traitement.

Dans ce contexte, les principaux objectifs d'une restauration sont d'améliorer les conditions de conservation de la dépouille et des éléments qui lui sont associés, car du point de vue technique, la restauration porte sur des matériaux organiques plus ou moins liés entre eux ; de viser à l'intégrité de l'individu en redonnant une cohésion au corps, c'est-à-dire, en réunissant les diverses parties du corps éventuellement dissociées ${ }^{15}$ et en replaçant, quand cela est possible, un élément détaché dans sa position anatomique (fig. $2 \mathrm{a}, \mathrm{b})^{16}$; et
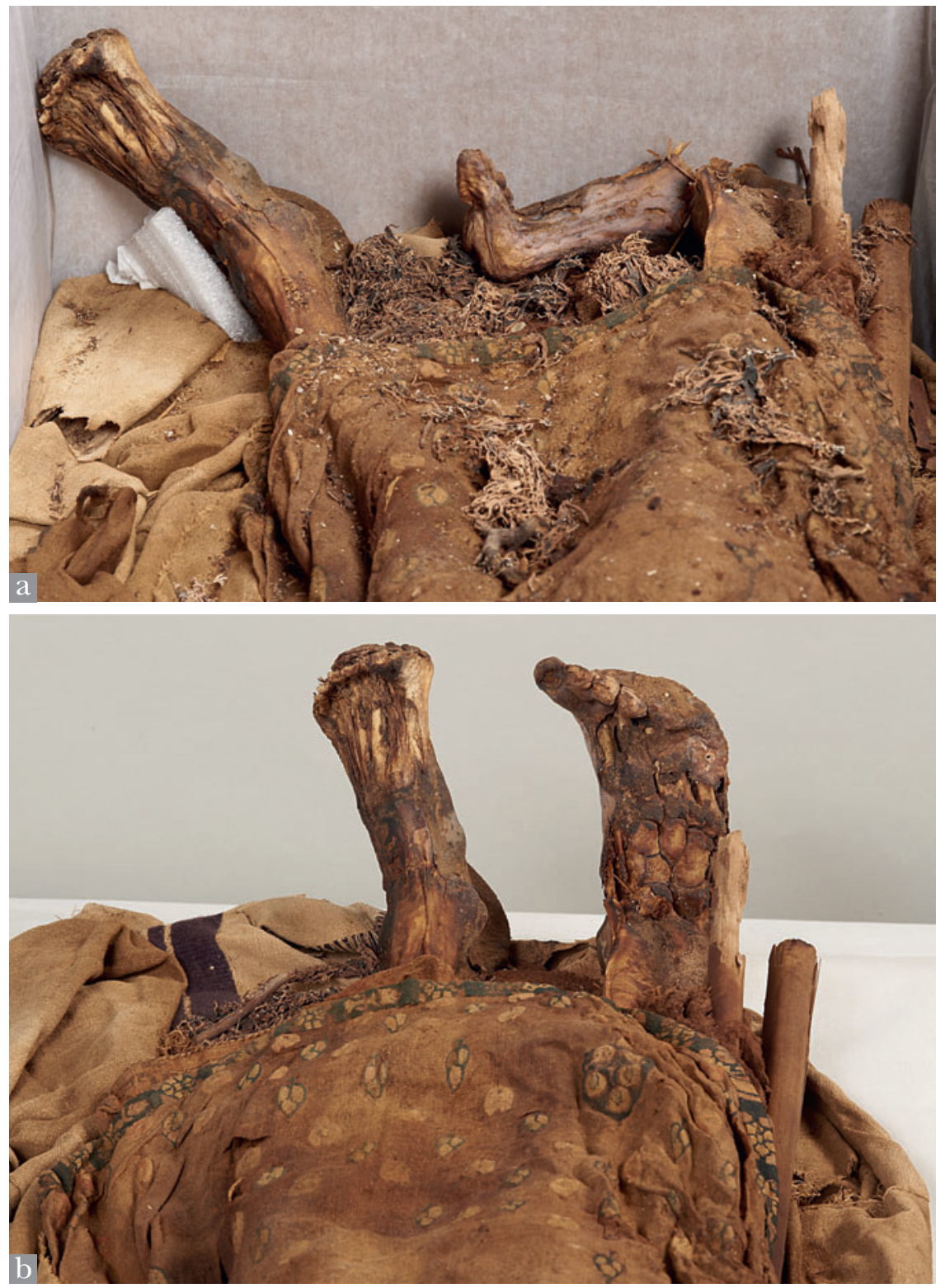

Fig. $2 a$, b. Restitution du pied dans sa position anatomique afin de redonner l'intégrité à la dépouille. Corps desséché copte, musée Joseph-Denais, Beaufort-en-Vallée. 


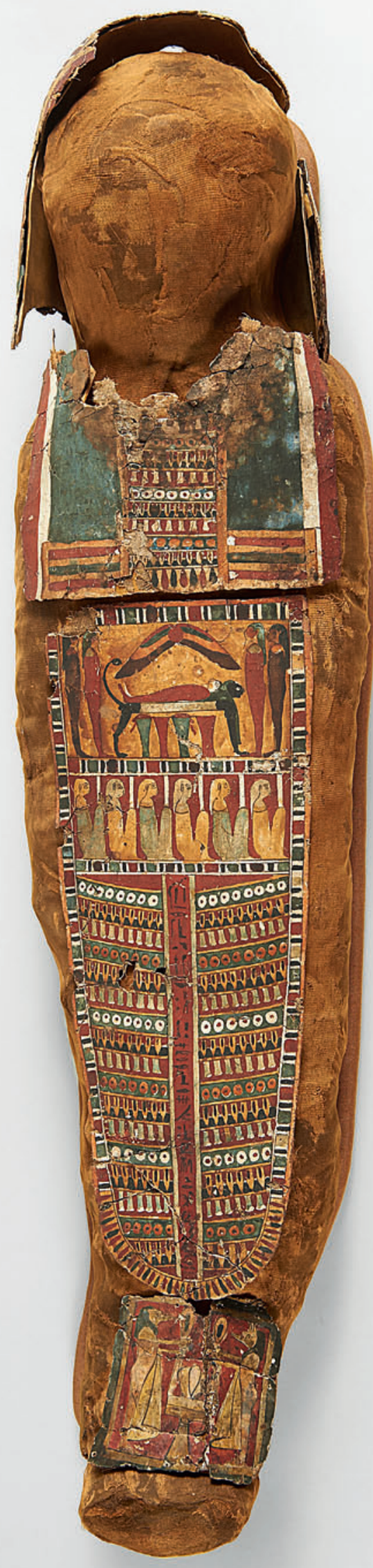

de favoriser une lisibilité maximale dans la perspective de l'exposition (momie dans son cercueil par exemple ; restitution des pieds manquants pour améliorer la compréhension du public ou reprise des collages de fragments d'une tête réduite qui nuisent à sa perception) tout en s'attachant à appliquer un traitement réversible et respectueux de l'innocuité et de la stabilité des matériaux employés. La démarche se cantonne souvent à des traitements de stabilisation et de remise en place par des moyens mécaniques, le recours à des techniques de collage et de consolidation n'étant envisagé qu'en dernier ressort si aucune alternative ne peut être trouvée. Et dans ce cas, l'introduction d'adhésif est limitée à la zone où la peau soulevée risque de se détacher ou d'être arrachée par exemple. Une méthode de doublage avec l'emploi de papier japonais peut être choisie, supposant un apport limité en adhésif et une adaptation du papier de doublage à la surface et aux déformations du corps sans exercer aucune contrainte tout en consolidant; et pouvant être facilement retiré. Ainsi, une plus grande résistance est apportée aux zones fragiles et soulevées, évitant toute perte de matière qui est alors stabilisée et permet des manipulations, dans le cadre des études notamment.

Il faut également prendre en considération les éléments humains isolés (tête, main, pied), nombreux dans les collections, et pour lesquels un nouveau support permet d'optimiser la conservation. La réalisation d'une boîte de conservation avec plateau de manipulation permet alors de sécuriser ces fragments de corps.

Les restes humains sont composés de matière organique, particulièrement périssable et fragile, sensible aux variations d'humidité, de température et d'éclairement. Par conséquent, leur traitement suppose une approche similaire à tout autre objet constitué de ce même matériau. Aussi, du point de vue de la restauration, l'intervention ne diffère pas beaucoup des autres objets en matériaux organiques. Mais le restaurateur n'est pas confronté à un objet comme un autre, il est face à un sujet, un individu, ce qui n'est pas neutre. Les solutions adoptées seront donc différentes, tout comme la façon de procéder : retenue, degré d'intervention limité, etc. Le niveau d'intervention sera variable et le traitement toujours envisagé en évaluant les risques éventuels. Bien souvent, ce sont les éléments périphériques, tels que les textiles, cartonnages, éléments de parure, etc. qui font l'objet du traitement (fig. 3). Mais parfois, le corps pourra être directement concerné, ce qui suppose des choix d'intervention plus délicats avec des questionnements quant aux limites qui en découlent : comment, par quels moyens, jusqu'où doit-on intervenir ? Parfois il n'est pas envisageable de supprimer un ancien soclage aujourd'hui considéré comme trop intrusif, car le risque est d'altérer davantage le corps. Si un élément métallique, tels les crochets qui servaient à suspendre un corps pour sa

Fig. 3. Intervention indirecte sur le corps : traitement des cartonnages et des textiles, momie d'enfant, musée d'Histoire locale,

Rueil-Malmaison. (c) C2RMF/A. Chauvet. 
présentation, ne peut être retiré sans altérer le squelette ou la peau, il sera conservé par exemple. Des solutions alternatives seront alors privilégiées ${ }^{17}$. Quand on ne peut plus rien faire en termes d'intervention, bien souvent la conservation préventive reste la seule solution. Il s'agira avant tout de limiter les risques de dégradation. On joue alors sur les paramètres de conservation. C'est le cas d'une tête de momie pulvérulente du musée de Châteaudun. Trop dégradée et ne pouvant plus être présentée car ne pouvant plus illustrer le propos scientifique de l'exposition, elle a été placée dans les réserves.

Toute intervention dépendra donc du contexte, du vestige, mais aussi de la sensibilité individuelle du restaurateur. Le plus souvent, un compromis sera choisi, visant à limiter le risque de dégradation ayant pu être engendré par d'anciennes pratiques, des facteurs environnementaux ou humains.

\section{Conclusion}

La conservation-restauration des restes humains pose de nombreuses questions. "S'il peut être considéré comme élément d'une collection, [le corps humain] appelle une réflexion particulière (...) postée sur le terrain de l'éthique et, partant, d'éventuelles normes déontologiques puisées à ce ressort ${ }^{18}$. " Les corps humains patrimonialisés constituent des cas particuliers, étant donné la variabilité des mécanismes et les degrés de préservation qui peuvent se rencontrer, mais aussi la relativité de la notion d'altération qui conditionne la démarche de conservation.

Par conséquent, tout traitement de restauration doit toujours être envisagé en évaluant au préalable sa portée et les risques éventuels. De nombreux compromis sont alors nécessaires et les questionnements permanents afin de ne pas corrompre les informations issues du corps. Certaines restaurations peuvent être jugées comme trop invasives. Il conviendra alors d'être prudent, et de ne pas chercher à tout prix à restaurer. Les protocoles seront établis en fonction des objectifs et des usages. Ainsi, un usage scientifique impliquera de porter davantage attention à l'intégrité de la matière pour conserver le potentiel informatif tandis qu'un usage pour une exposition visera avant tout l'aspect visuel. Pour aider aux choix, il faut intégrer la dimension humaine dans la démarche de la conservation-restauration, soit le respect vis-à-vis de l'individu, le principe de précaution sans oublier la dignité. Ces composantes et les règles éthiques sous-jacentes introduisent des limites dans les moyens matériels de conservation à disposition. Ainsi, lorsque les cheveux d'un corps nécessitent une intervention pour être «consolidés » au cuir chevelu, il importe de s'interroger sur le potentiel informatif qu'ils représentent. Si la totalité du support est concerné, le choix pourra être fait de ne pas consolider, car l'ensemble du potentiel informatif sera touché par le produit employé ${ }^{13}$. C'est également le cas pour l'ADN ancien qui suppose des précautions particulières pour en assurer l'étude. Il importe de garantir sa conservation pour les futures recherches qui pourront être menées avec le développement des technologies.

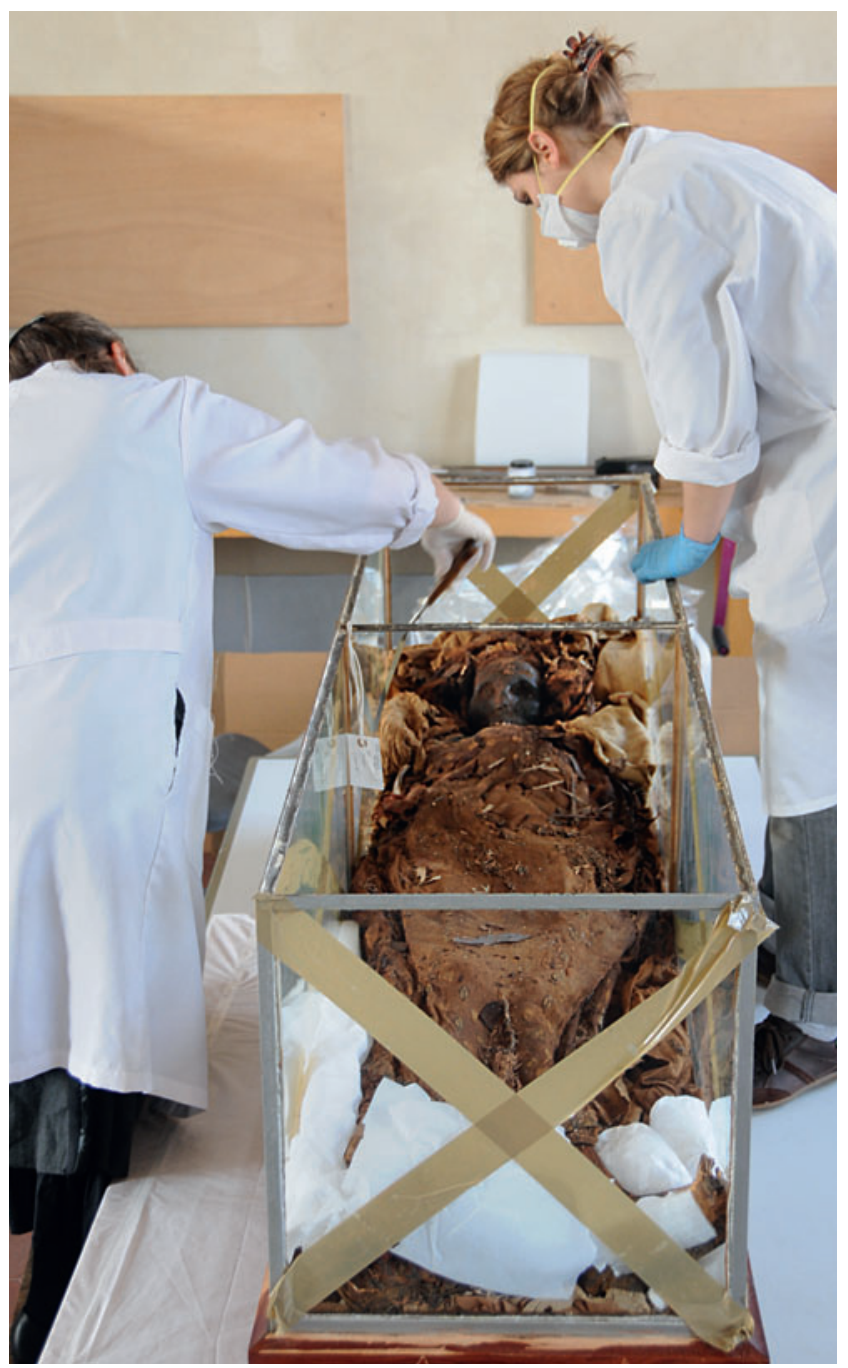

Fig. 4. Restaurateurs spécialisés travaillant en commun sur une momie égyptienne. (c) C2RMF/G. de Puniet.

De fait, il faut avant tout une adéquation entre le besoin, le risque et l'intérêt de la restauration avec la déontologie. Par conséquent, les objectifs de la restauration doivent être clairement définis ainsi que l'objet de la restauration : la matière ou la technique au service de la monstration comme les préparations anatomiques, mais aussi s'il y a le risque de pertes d'informations au cours de l'opération de restauration ${ }^{1}$. Les limites techniques doivent aussi être prises en compte car souvent certaines altérations sont irréversibles.

Quoi qu'il en soit, il importe que les considérations de conservation-restauration soient prises en compte à tous les niveaux (fouille, exposition, étude) afin de participer à une démarche plus respectueuse.

La déontologie de la conservation-restauration appliquée au domaine des restes humains implique par conséquent l'intervention de restaurateurs spécialisés développant une pratique appropriée à la spécificité de ces collections (fig. 4). 


\section{Notes}

1. L'éthique est la science des règles qui gouvernent les comportements humains ; et ce qui concerne la morale. Tandis que la déontologie est l'ensemble des règles et des devoirs moraux qui s'imposent à un professionnel dans l'exercice de son métier (voir Bergeon Langle et Brunel, 2014, p. 169170 et p. 133-135).

2. Cadot, 2009 ; Cadot, 2007, p. 4-15; Cadot, 2012, p. 3-11 et Cadot, 2015, p. 32-34.

3. Voir également Ameisen et Coz, 2010 ; Novljanin Grignard, 2012.

4. De même, la mise en place d'un cours de sensibilisation pour les conservateurs à l'Institut national du Patrimoine, d'un séminaire de master à l'École du Louvre, ainsi que le développement de mémoires de recherche sur ce sujet et la formation de restaurateurs spécialisés (Cadot, 2015).

5. Ce groupe de travail conduit par M. Van Praët a pour objectifs d'établir une cartographie et la typologie des collections publiques françaises conservant des restes humains en vue d'élaborer un guide de gestion professionnelle respectueuse de la dignité humaine et d'éléments de doctrine en matière d'éventuelles restitutions. Il doit également réfléchir au statut juridique des restes humains dans les collections et à leur exposition (voir Rapport au Parlement prévu par l'article 4 de la loi n ${ }^{\circ}$ 2010-501 du 18 mai 2010 et annexe 14).

6. Department for Culture, Media and Sport, 2004 et 2005 ; The British Museum Policy on Human Remains (en ligne).

7. Voir également à ce sujet la réflexion sur le rôle des restaurateurs (Cassman et Odegaard, 2004).

8. European Confederation of Conservator-Restorers' Organisations (ECCO) Guidelines (I), 2002.

9. L'article 9 du code de l'ECCO précise que « le Conservateur-Restaurateur doit chercher à n'utiliser que des produits, matériaux et procédés qui, correspondant au niveau actuel des connaissances, ne nuiront pas aux biens culturels ni à l'environnement et aux personnes. L'intervention et les matériaux utilisés ne doivent pas compromettre, dans la mesure du possible, les examens, traitements et analyses futures. Ils doivent également être compatibles avec les matériaux constitutifs du bien culturel et être, si possible, facilement réversibles ».

10. Les restes humains qui sont parvenus jusqu'à nous l'ont été selon divers contextes. Ils ont pu être conservés de façon fortuite dans un environnement qui a favorisé leur préservation (climat, nature du sol, etc.). Ou, au contraire, ils ont subi une préparation spécifique, intentionnelle (éviscération, emploi de baume, d'onguents, etc.) dont le but était de les préserver de la putréfaction.

11. Articles 2.5, 3.7 et 4.3 .

12. Le principe de dignité est rappelé dans le Code pénal et le Code civil français, ainsi que dans les lois relatives à la Bioéthique (article 16-1-1 du Code civil introduit par la loi n 2008-1350 du 19 décembre 2008; Loi Bioéthique n 94-653 du 29 juillet 1994 relative au respect du corps humain)

13. Carminati, 2010
14. Pour Fr. Delaporte (2014, p. 83), la restauration des restes humains est compliquée par le fait qu'une ambivalence est laissée entre l'objet à restaurer et la personne, entre la dépouille qu'il faut traiter avec dignité et la personne. Pour lui, « (...) si nous nous laissons aller à sacraliser les restes en y logeant la "personne humaine" on oublie l'essentiel : à savoir, d'une part les restes à conserver et, de l'autre, la restitution des significations culturelles dont ils sont les supports ».

15. La tête de la momie du musée Annede-Beaujeu à Moulins-sur-Allier a ainsi été rapprochée de son corps duquel elle avait été séparée lors de l'opération de débandelettage. Les connexions anatomiques étant rompues, il n'était pas possible de raccrocher physiquement la tête et le corps sans risque d'altérer l'une ou l'autre partie. Les restaurateurs ont donc réalisé un support spécifique en matériau neutre pour la tête, évitant ainsi les manipulations directes et favorisant son positionnement au niveau de l'espace dévolu à la tête dans le cercueil. Ainsi, aucun adhésif risquant de compromettre de futures investigations ou autre matériau n'a été utilisé (Bèche-Wittmann et al., à paraître).

16. Cadot et al., 2014.

17. Des mesures de prévention sont souvent privilégiées pour agir en amont, sur les causes de dégradations. La conservation préventive constitue alors le meilleur moyen pour préserver ces corps.

18. Cornu, 2012, p. 2.

\section{Bibliographie}

Ameisen J.-Cl. et Le Coz P. (rapporteurs), Avis $n^{\circ} 111$ : avis sur les problèmes éthiques posés par l'utilisation des cadaures à des fins de conservation ou d'exposition muséale, Paris, Comité consultatif national d'éthique pour les sciences de la vie et de la santé, 7 janvier 2010, 15 p., [en ligne] URL: http://www.ccneethique.fr/docs/ Avis_\%20111.pdf

Bèche-Wittmann M., Blaise V., Carminati P., Henon J. et Timbart N., « Étude et conservation d'une momie égyptienne anonyme de la XXII ${ }^{\mathrm{e}}$ dynastie. Musée départemental Anne-de-Beaujeu, Moulins-sur-Allier, in la Revue des musées de France, à paraître.

Bergeon Langle S. et Brunel G., 2014 La restauration des œuvres d'art. Vade-mecum en quelques mots, Hermann.

Cadot L., 2009, En chair et en os : le cadavre au musée. Valeurs, statuts et enjeux de la conservation des dépouilles humaines patrimonialisées, École du Louvre/RMN, Paris.

Cadot L., 2007, « Les restes humains : une gageure pour les musées ? ", La Lettre de l'OCIM, n ${ }^{\circ} 109$, p. 4-15.

Cadot L., 2012, « La conservation-restauration des restes humains en question ", Coré, n 27 , p. 3-11.

Cadot L., 2015, « Actualité récente et évolution des pratiques autour des restes humains patrimonialisés ", La Lettre de l'OCIM, $n^{\circ} 157$, p. $32-34$.

Cadot L., Coudert M., Dal Prà P., Gandolfo N., Langlois J., Richardin P., Timbart N. et
Weygand S., 2014, «Étude et restauration de deux momies du musée Joseph-Denais de Beaufort-en-Vallée », Technè n 38 , p. 58-64.

Cassman V. et Odegaard N., 2004, "Human remains and the conservator's role", Studies in conservation, $\mathrm{n}^{\circ} 49$, p. 271-282.

Cassman V., Odegaard N. et Powell J., 2007, Human Remains: Guide for Museums and Academic Institutions, AltaMira Press.

Code de déontologie de l'ICOM pour les musées, 2006

Cornu M., 2012, « Exposer le corps humain », Coré, $\mathrm{n}^{\circ} 27$, p. 2

CSNC, Rapport au Parlement de la Commission Scientifique Nationale des Collections (Contributions aux travaux de la CSNC), fascicule $n^{\circ} 3$, annexe $n^{\circ} 14$, Contribution du groupe de travail sur les restes humains, p. 97-116.

Delaporte Fr., 2014, «Les rapports de l'homme avec les restes humains aujourd'hui ", Support Tracé, $\mathrm{n}^{\circ} 14$, p.79-84.

ICOM Code of Ethics for Natural History Museums, 2013

Department for Culture, Media and Sport, 2004, Care of Historic Human Remains. A consultation on the Report of the Working Group on Human Remains, DCMS, Londres.

Department for Culture, Media and Sport, 2005, Guidance for the care of Human Remains in Museums, DCMS, Londres.

Des collections anatomiques aux objets de culte: conservation et exposition des restes humains dans les musées, Actes du colloque, musée du quai Branly, 22-23 février 2008 [en ligne] URL : http://www.quaibranly.fr/ fileadmin/user_upload/pdf/Version_ Francaise_SymposiumRestes_Humains. pdf

ECCO (Confédération européenne des organisations des conservateursrestaurateurs), 2003, La profession de conservateur-restaurateur, code d'éthique et formation.

Fletcher A., Antoine D. et Hill J.D., 2014 Regarding the dead: Human remains in the British museum, British Museum Research Publication, $\mathrm{n}^{\circ} 197$

Novljanin Grignard I. N., 2012, « Les musées et leurs restes humains : différents regards et nouveaux enjeux ", in Les cahiers du musée des Confluences, Études scientifiques, $\mathrm{n}^{\circ} 3$, p. 11-16.

The British Museum Policy on Human Remains, London, The British Museum; [en ligne] URL : http://www.thebritishmuseum. ac.uk/PDF/HumanRemains.pdf

\section{Document inédit}

Carminati P., 2010, Étude et conservation. Problématiques de la restauration d'une momie andine des collections du musée de l'Homme: Momie de femme adulte. Région de Tarapaca, Chili, XI siècle-1re moitié du XII siècle. Ancienne collection anthropo-logique du Muséum d'histoire naturelle, Paris. Mémoire de diplôme de conservation-restauration des œuvres sculptées, École supérieure des Beaux-arts de Tours, sous la direction de Anne Raggi, inédit. 